\title{
Dynamics of relativistic electrons in the region of outer radiation belt, caused by solar events
}

\author{
Koldashov S.V. * \\ Author affiliation \\ National Research Nuclear University MEPhI (Moscow Engineering Physics Institute) \\ E-mail: SVKoldashov@mephi.ru
}

\author{
Aleksandrin S.Yu. \\ Affiliation \\ National Research Nuclear University MEPhI (Moscow Engineering Physics Institute) \\ E-mail: SYAleksandrin@mephi.ru \\ Eremina N.D. \\ Affiliation \\ National Research Nuclear University MEPhI (Moscow Engineering Physics Institute) \\ E-mail: Eremina.Nadlen@yandex.ru
}

\begin{abstract}
The results of the observation of short-term and long-term variations of relativistic electron flux in the region of outer radiation belt with ARINA and VSPLESK satellite experiments are presented. Scintillation spectrometers ARINA (on board the Resurs-DK1 Russian satellite, since 2006) and VSPLESK (on board the International Space Station, since 2008) provide continuous measurements of high-energy particle fluxes in low-Earth orbits, detect and identify electrons and protons with energies in 3-30 MeV and 30-100 MeV ranges correspondingly, give the possibility to study energy spectra, pitch-angle distributions and time profiles of particle fluxes. Detail analysis of experimental data on relativistic (4-6 MeV) electrons obtained in 2012 was fulfilled. It was revealed a large variability of electron flux in zone of the outer radiation belt. Analysis has shown that observed effects in electron flux were caused by solar-magnetosperic disturbances.
\end{abstract}

The 34th International Cosmic Ray Conference,

30 July- 6 August, 2015

The Hague, The Netherlands

${ }^{*}$ Speaker. 


\section{Introduction.}

As it is well known from earlier satellite experiments, the outer radiation belt is very high dynamic formation [1] and references therein. Some mechanisms of relativistic electron belt generation and loss are considered in [2], [3]. Latest measurements of relativistic electron flux in the outer radiation belt, fulfilled by two Van-Allen probes in high apogee orbit, gave new very important information about the behavior of high-energy electronensemble in the outer belt [4]. On the basis of this observation it is asserted that there is the local structure of outer radiation belt with a strong variability. In particular it was noticed that the third radiation belt was formed in the outer magnetosphere at the beginning of September 2012. Two Russian satellite experiments, aimed to study fast variations of high-energy electron and proton fluxes in the magnetosphere, are carried out in low Earth orbit since 2006 [5]. Results concerning the observation of relativistic electron flux in the zone of outer radiation belt at 3-3.5 L-shell range in 2012 are presented below.

\section{Instruments.}

The ARINA and VSPLESK scintillation spectrometers developed by the MEPhI are fully the same in physics schemes, have identical physical parameters (geometrical factor, energy range, energy resolution, and others), detect and identify electrons (3-30 MeV) and protons (30-100 MeV), measure particle energies, and allow to study the energy spectra and time profiles of particle fluxes.

The ARINA and VSPLESK experiments are carried out on board the low-orbit spacecrafts. The ARINA instrument is installed in a hermetic container of the Resurs-DK1 satellite with an altitude of $350-600 \mathrm{~km}$ and an orbit inclination of $70^{\circ}$. The experiment is executed since the midJune, 2006 [6].

The VSPLESK instrument is installed outside the sealed volume on the International Space Station (altitude is $350-400 \mathrm{~km}$, an orbit inclination is $51^{\circ}$ ). Measurements are carried out since August, 2008) [7].

Local pitch-angles of particles during the measurements were close to $90^{\circ}$ for both experiments.

Figure 1 shows the layout of the multilayer scintillation detector (MSD) which is main part of the instruments. Charged particles (electrons, protons) moving forward and arriving at the instrument aperture sequentially pass through scintillation layers C1, C2, C3, etc., lose energy, and are absorbed in the MSD. Backward particles and particles passed through the entire instrument are cut by detector $\mathrm{C} 10$ operating in the anticoincidence mode. Thus, particles stopped in the MSD are electrons with energies of $3-30 \mathrm{MeV}$ and protons with energies of $30-100 \mathrm{MeV}$. They are identified by the energy release in each layer when passing through the instrument (MSD) and by the range in it. The electron and proton energy is measured by their range in MSD layers. The physics scheme and performances of the instrument are described in detail in [8].

The instruments give the possibility to measure the energy spectra of particles and to trace their evolution, to determine time profiles of particle flux variations with high time resolution, and can operate in high-intensity particle fluxes of the radiation belt. The instrument acceptance controlled by the configuration and arrangement of detectors $\mathrm{C} 1-\mathrm{C} 3$ is about $10 \mathrm{~cm}^{2} \cdot \mathrm{sr}$. 


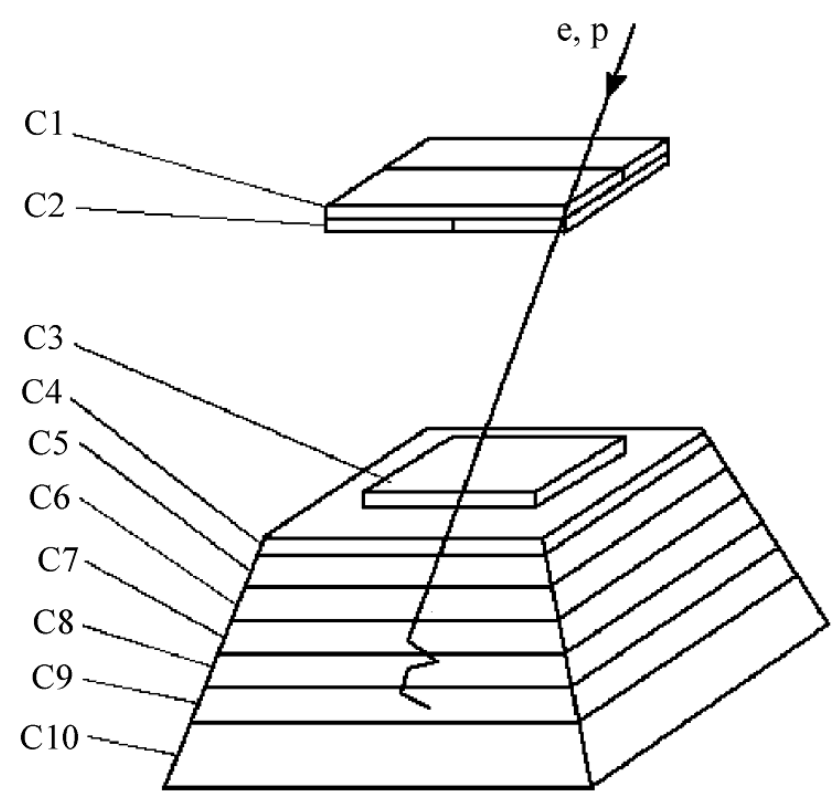

Figure 1: Physical diagram of the spectrometer.

The main objective of experiments is the study of high-energy charged particle bursts of solar and geophysical origin in the inner zone of magnetosphere [5].

For this work we used the ARINA and VSPLESK experimental data on the relativistic electron flux in the narrow energy range of $4-6 \mathrm{MeV}$ in the inner edge of outer radiation belt.

\section{Observation of high-energy electrons at 3 - 3.5 L-shells.}

In [9] analysis of variation of L-distributions of $4-6 \mathrm{MeV}$ electron flux in the outer radiation belt were carried out on the basis of ARINA and VSPLESK data for the beginning 2012. It was marked that there were quiet geomagnetic conditions in January and February, and so, low intense flux of relativistic electrons was observed. Series of powerful solar flares and CME in March 2012 gave rise to strong disturbance of the Earth's magnetosphere (Dst $\sim-130 \mathrm{nT}$ ) and $4-6 \mathrm{MeV}$ electron flux sharply increased an two orders of value. New additional unstable radiation belt of relativistic electrons was generated in the beginning of March at L-shells more 3. It was observed that this belt existed during several weeks and disappeared in the beginning of April 2012. It is necessary to mark that most sensitive to disturbance zone of $4-6 \mathrm{MeV}$ electrons was locates at $\mathrm{L}=3$ -3.5 .

For next detail analysis L shells in the narrow range 3-3.5 were chosen in our work. Figure 2 presents the map of 4-6 MeV electron flux at $\mathrm{L}=3-3.5$ observed in 2012. The figure illustrates the variability of this zone of outer radiation belt in high energy range of electrons. As it was noticed above (see Introduction) such a situation is usual and the main reason for that is the solar events (flares and CME) reducing to strong magnetospheric disturbances. From fig. 2 we see quiet conditions in January and low intense flux of relativistic electrons at chosen L shells.However series of powerful solar flares and CME in March 2012 changed the situation and gave rise to 

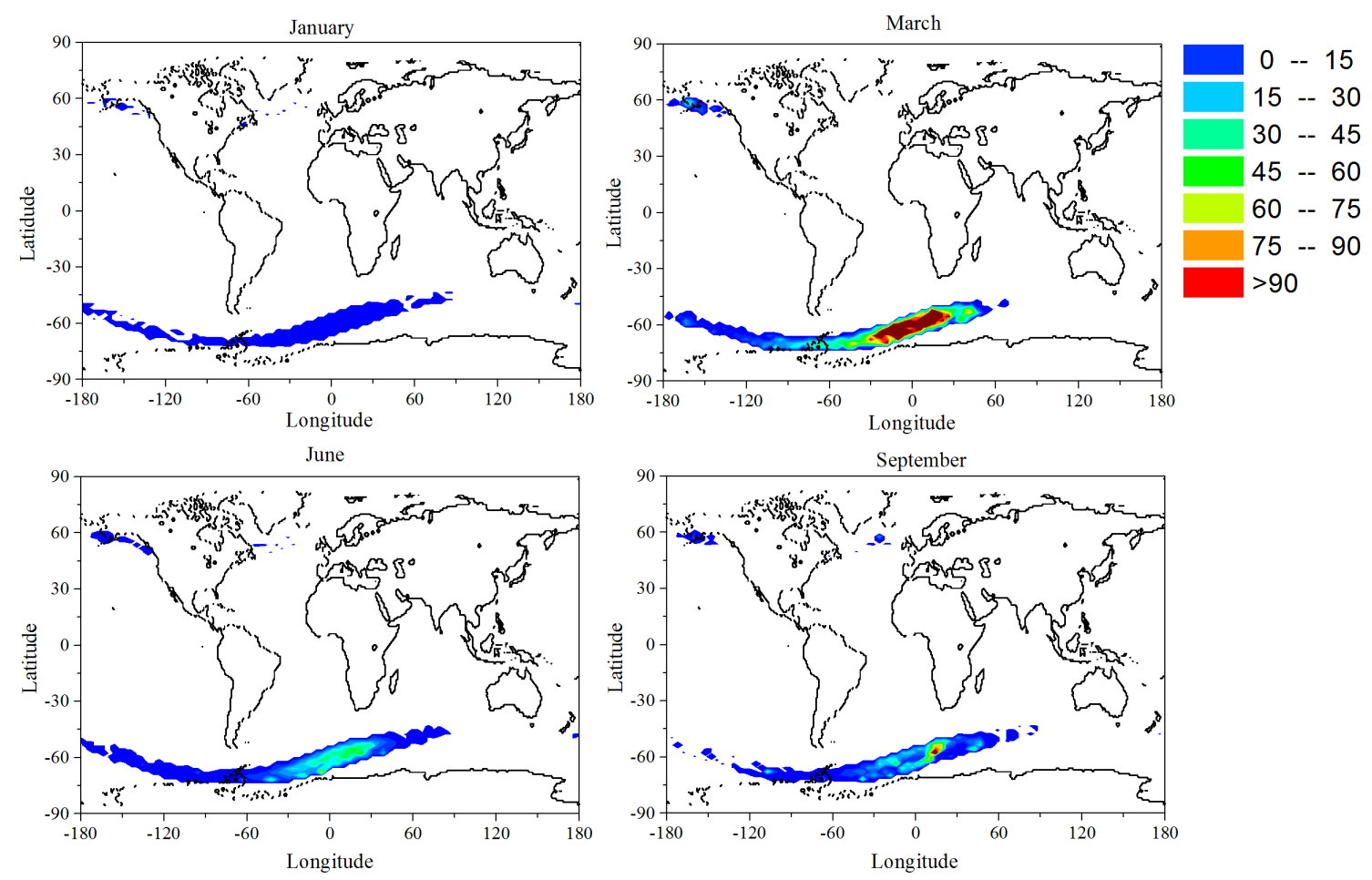

Figure 2: Geographical map of flux of $4-6 \mathrm{MeV}$ electrons at $3-3.5 \mathrm{~L}$ shells observed by ARINA in January, March, June and September 2012.

strong disturbance of the Earth's magnetosphere. As a result $4-6 \mathrm{MeV}$ electron flux sharply and significantly increased. These effects (solar events and particle flux growth) repeated several times in 2012.

Most detail data obtained by ARINA is presented in fig. 3. Arrows $(\mathrm{t} 1, \mathrm{t} 2, \mathrm{t} 3, \mathrm{t} 4)$ in the figure mark the sharp changing (grows or drop) in 4-6 MeV electron flux at $\mathrm{L}=3-3.5$. Each of these variations coincides with time of strong magnetospheric disturbances with Dst value about $100 \mathrm{nT}$.

Also it is necessary to mark that ARINA observation revealed that the strong geomagnetic storm is able to cause as sharp increase as sharp decrease of relativistic electron flux at chosen $\mathrm{L}$ shells.

\section{Discussion.}

We presented in this work one year ARINA and VSPLESK orbital measurements of relativistic electrons for narrow range of L shells (3-3.5) for the following reason. Since September two Van Allen probes began to search the variability of outer radiation belt and it was published about the discovery of third radiation belt in the magnetosphere [9]. Four months 2012 are the same for measurements of relativistic electrons by ARINA, VSPLESK and Van Allen probes. Taking into account the differences in orbits of satellites it is possible in principle to compare the behavior of relativistic electrons in two different zones of the same range of $L$ shells. First zone contains the particles with mirror points at the top of L shells (Van Allen probes) and the second one contains the particles with mirror points at the foot of L shells (ARINA and VSPLESK). Obviously, these 


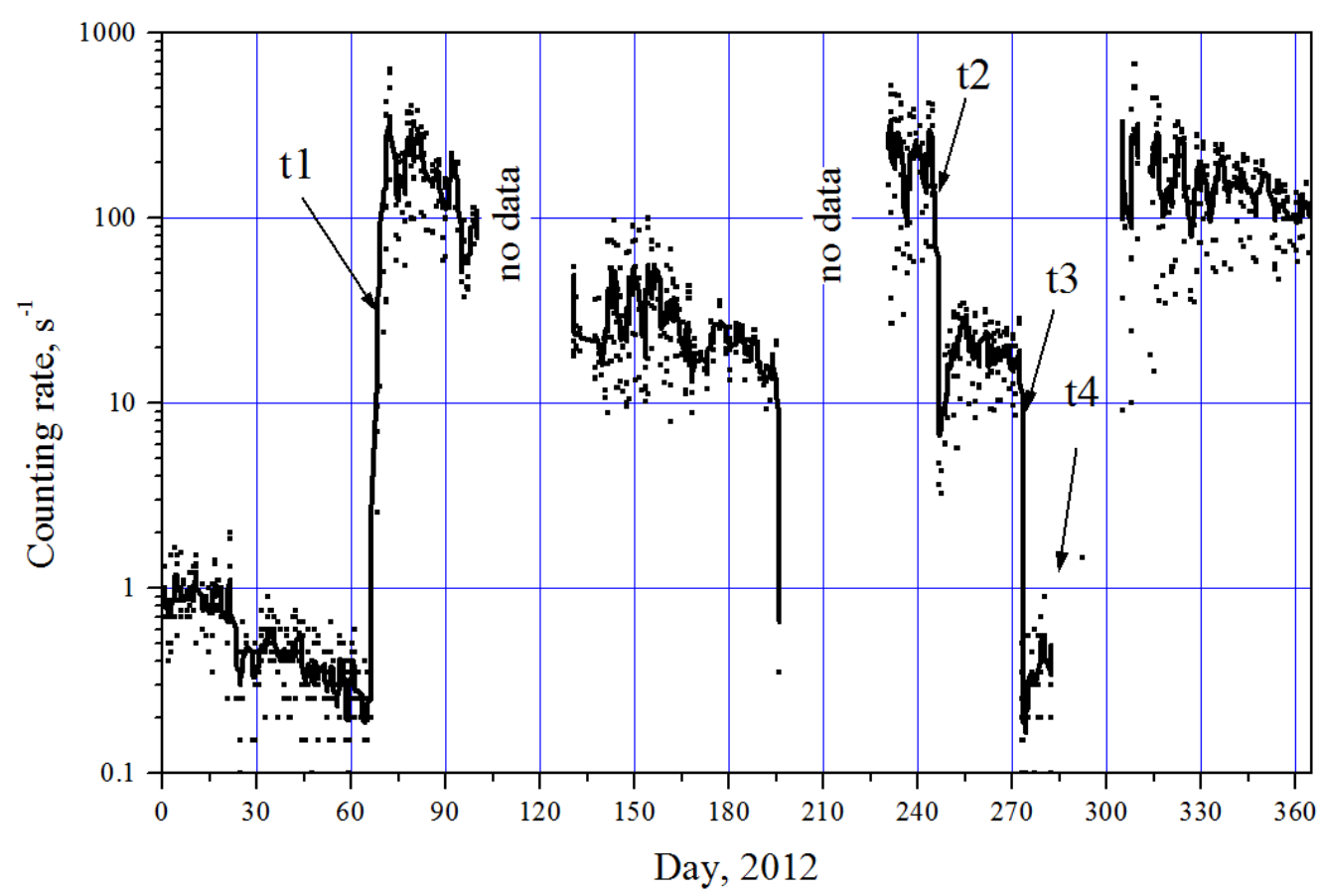

Figure 3: Variation of flux of $4-6 \mathrm{MeV}$ electrons at 3 - $3.5 \mathrm{~L}$ shells observed by ARINA in 2012. Points in the figure is counting rates of electrons in $20 \mathrm{~s}$ intervals. Curve is approximation of experimental data.

two different groups of electrons have different equatorial pitch-angles, and so it is possible to study experimentally the efficiency of particle-wave interactions in different conditions.

\section{Conclusion.}

Variations of 4-6 MeV electron flux at $\mathrm{L}=3-3.5$ in the outer radiation belt were studied with ARINA and VSPLESK experiments. It was shown the strong variability of the flux of these particles during the geomagnetic storms caused by powerful solar events.

\section{References}

[1] J.F. Lemaire, D. Heiderics, and D.N. Backer, Editors. Radiation belts: Models and Standards, Geophysical Monograph 97. American Geophysical Union. 1997. 322 p.

[2] Lyons, L.R., Thorne, R.M. and Kennel, C.F. Electron pitch-angle diffusion driven by oblique whistler-mode turbulence. J. Plasma. Phys.. V 6, p.589-606 (1971).

[3] Shprits, Y.Y. and Thorne R.M. Dynamic evolution of energetic outer zone electrons due to wave-particle interactions during storms. J. Geophys. Res. 112, 2156-2202 (2007).

[4] D.N. Backer, S.G. Kanekal, V.C. Hoxie, M.G. Henderson, X. Li, H.E. Spence, S.R. Elkington, R.H.W. Friedel, J. Goldstein, M.K. Hudson, G.D. Reeves, R.M. Thorne, C,A. Kletzing, S.G. Claudepierre. A long-lived relativistic electron storage ring embedded in Earth's outer Van Allen belt. Science. 12 April 2013. Vol. 340, p. 186-190. 
[5] S. Yu. Aleksandrin, A. V. Bakaldin, A. G. Batischev, M. A. Bzheumikhova, A. M. Galper,S. V. Koldashov, A. A. Ulitin, and N. D. Sharonova. Local Disturbances of Lightning and Seismic Origin in the Radiation Belt. Bulletin of the Russian Academy of Sciences. Physics, 2013, Vol. 77, No. 5, pp. 578âĂŞ580.

[6] A.V. Bakaldin, A.G. Batischev, A.M. Galper, L.A. Grishantseva, S. V. Koldashov, P. Yu. Naumov, V. Yu. Chesnokov, and V. A. Shilov. . Cosmic Research. Vol 45. No. 5. p. 471 (2007).

[7] S. Yu. Aleksandrin, A. V. Bakaldin, A. G. Batischev, M. A. Bjeumikhova, S. A. Voronov, A. M. Galper, L. A. Grishantseva, S. V. Koldashov, P. Yu. Naumov, V. Yu. Chesnokov, N. D. Sharonova, and V. A. Shilov. Izvestiya Academiy Nauk, physics series. Vol 73, âĎ́́ 3, p. 379 (2009).

[8] A. V. Bakaldin, A. G. Batischev, M. A. Bjeumikhova, S. A. Voronov, A. M. Galper, L. A. Grishantseva, S. V. Koldashov, P. Yu. Naumov, V. Yu. Chesnokov, and V. A. Shilov.. Instrumentation for Experimental Recording of Bursts of High-Energy Charged Particles in Near-Earth Space, Izv. Akad. Nauk, Ser. Fiz., 2005, vol. 69, no. 6, p. 918.

[9] S.Yu. Aleksandrin, A.V. Bakaldin, A.G. Batischev, M.A. Bjeumikhova, A.M. Galper, L.A. Grishantseva, S.V. Koldashov, N.D. Sharonova, P.I. Solodskikh, A.A. Ulitin, T.R. Zharaspaev. High-energy charged particle flux dynamics in the near-Earth space caused by solar-magnetospheric and geophysical phenomena. Proc. 33rd International Cosmoc ray Conference, Rio de Janeiro 2013. 0882-2013. 